\title{
Taxonomy Formation by Approximate Equivalence Relations, Revisited
}

\author{
F.A. El-Mouadib ${ }^{1}$, J. Koronacki ${ }^{1,2}$, and J.M. Żytkow ${ }^{1,3}$ \\ 1 Institute of Computer Science, Polish Academy of Sciences, \\ 2 Polish-Japanese Institute of Computer Technology \\ ${ }^{3}$ Computer Science Department, UNC Charlotte \\ mouadib, korona@ipipan.waw.pl, zytkow@uncc.edu
}

\begin{abstract}
Unsupervised classification of objects involves formation of classes and construction of one or more taxonomies that include those classes. Meaningful classes can be formed in feedback with acquisition of knowledge about each class. We demonstrate how contingency tables can be used to construct one-level taxonomy elements by relying only on approximate equivalence relations between attribute pairs, and how a multi-level taxonomy formation can be guided by a partition utility functions. Databases with different types of attributes and large number of records can be dealt with.
\end{abstract}

\section{Introduction}

As a part of their 49er system, Żytkow and Zembowicz (1993, 1995) and Troxel et al. (1994) proposed a mechanism for taxonomy formation based on approximate equivalence relations in two-way contingency tables.

In this paper, we limit our attention to construction of binary taxonomy trees. At each node of the tree, only a binary split is allowed. Alike 49er, our mechanism starts from one-level taxonomies. A systematic search is performed for pairs of attributes whose association is "strong enough" in a well defined sense. If such approximately equivalent pairs are found at a particular node of the tree, one of one-level taxonomies derived from approximate equivalence is added under this node.

In what follows, we deal with some modifications and extensions of the original methodology of Żytkow and others. In particular,

1. due to their simplicity and arguably clear interpretation, measures of association introduced by Goodman and Kruskal (1954) are used in lieu of $\chi^{2}$-like Cramer's $V$ statistics applied in 49er (Troxel et al., 1994; Żytkow and Zembowicz, 1996).

2. instead of using the original heuristic approach intended to minimize the description length of the taxonomy, the choice of a one-level taxonomy to be attached under a given node is based on maximization of the so-called partition utility (as suggested, e.g., by Fisher and Hapanyengwi (1993) and based either on entropy or on the Gini index); 
3. a data-driven discretization of continuous variables is proposed. The original system uses discretization based on equal intervals or any other user-defined discretization of attributes.

Given the system's algorithmic simplicity it is suitable for data mining applications. In the process of building a taxonomy tree, the system does not use any similarity/dissimilarity measures but instead it relies only on approximating equivalence relations and on using partition utility functions. It should also be emphasized that, like 49er, the system works in a strictly unsupervised fashion. No a priori model of the data is assumed (neither the number of classes is prespecified nor any assumptions about data distribution are made).

\section{Approximate Equivalence Relations}

Let us begin with a pair of attributes measured on a nominal scale. A two-way contingency table represents the equivalence relation between the two attributes involved if and only if both attributes can be binned so that the entire population is concentrated in cells no two of which are in the same row or column of the table. In real world databases, relations represented by such tables are rare. Therefore, like 49er, our system relies on approximate equivalence relations. But rather than comparing observed cell counts with the expected counts under independence to determine whether a contingency table approximates equivalence, as is the case when $\chi^{2}$-like statistics are used, we use a set of lambda $(\lambda)$ measures of association introduced by Goodman and Kruskal (1954)

Following Goodman and Kruskal (1954), let $A$ and $B$ be two nominal attributes assuming "levels", that is, values $A_{a}, a=1, \ldots, \alpha$, and $B_{b}, b=1, \ldots, \beta$, respectively. Consider first the following asymmetric case: the classification of the values of $A$ precedes the $B$ classification chronologically, causally, or otherwise. An individual is chosen at random from the population and we are asked to guess its $B$-level as well as possible, either

1. Given no further information, or

2. Given the individual's $A$-level.

One can then define the following measure of association

$$
\lambda_{b}=\frac{\text { (Probab. of wrong guess in case } 1)-(\text { Probab. of wrong guess in case } 2)}{(\text { Probab. of wrong guess in case } 1)} .
$$

It is easy to see that: $\lambda_{b}$ is indeterminate if and only if attribute $B$ assumes the same level for all the population (i.e., iff the population lies in one column of the contingency table); $\lambda_{b} \in[0,1] ; \lambda_{b}=0$ if and only if knowledge of the $A$ classification is of no help in predicting the $B$ classification; $\lambda_{b}=1$ if and only if the $A$ classification completely specifies the $B$ classification; if $A$ and $B$ are independent and $\lambda_{b}$ is determinate, then $\lambda_{b}=0 ; \lambda_{b}$ is unchanged by permutation of rows or columns. 
The $\lambda_{a}$ measure can be defined analogously. In turn, consider a symmetric case when an individual is chosen at random and the problem is to guess its $A$ value half the time and its $B$ value half the time (at random), either:

1. Given no further information, or

2. Given the individual's $A$-level when $B$ level is guessed and vice versa.

Clearly, the measure $\lambda$ can now be defined in the same way as $\lambda_{b}$. Both measures have analogous properties: $\lambda$ is indeterminate if and only if the population lies in one cell of the contingency table; $\lambda \in[0,1] ; \lambda=1$ if and only if all the population is concentrated in cells no two of which are in the same row or column of the table; if $A$ and $B$ are independent and $\lambda$ is determinate, then $\lambda=0 ; \lambda_{b}$ is unchanged by permutation of rows or columns; $\lambda$ lies between $\lambda_{a}$ and $\lambda_{b}$ inclusive.

The computation of $\lambda_{a}, \lambda_{b}$ and $\lambda$ is extremely simple. In particular, in terms of cell counts,

$$
\lambda=\frac{\sum_{a} n_{a m}+\sum_{b} n_{m b}-n_{\cdot m}-n_{m} .}{2 n-\left(n_{\cdot m}+n_{m \cdot}\right)},
$$

where $\mathrm{n}$ is the size of the population (total count), $n_{a m}$ is the maximum cell count in row $a, n_{m b}$ is the maximum cell count in column $b, n_{\cdot m}=\max _{b}\left\{\sum_{a} n_{a b}\right\}$ and $n_{m} .=\max _{a}\left\{\sum_{b} n_{a b}\right\}$. See Goodman and Kruskal (1954) and Bishop et al. (1975) for a detailed discussion of lambda measures, as well as for the definitions and a general treatment of other measures of association.

A cursory look at the properties of lambda measures shows that they are very well suited to measure not only association between nominal attributes but also their "closeness" to equivalence. In the nominal case, the strength of association and closeness to equivalence can be considered as essentially the same.

For attributes measured on an ordinal scale, association between them, can be represented by probabilities for like and unlike orders (positive or negative association). On the other hand, the issue of (approximate) equivalence is not tied to any notion of order. Thus, although the $\lambda$ measures are designed specifically to measure association when no order is present, they can be used to evaluate approximate equivalence between any ordered and nominal attributes.

Usually, clusters which are present in data described by ordered attributes reflect the ordered structure of the data: say, "small" values of an attribute appear in one cluster and "medium and large" values in another, not "small and large" in one and "medium" in another. While in this paper we stick to the lambda measures, in El-Mouadib and Koronacki (1999) a measure of closeness to equivalence based on probabilities of like and unlike orders is proposed (that other measure is a minor modification of Goodman and Kruskal's (1954) $\gamma$ measure of association of ordered attributes).

In summary, in order to form one-level taxonomies, a systematic search is performed for pairs of attributes whose strength of association is above some controllable threshold value. In our implementation, a threshold can be set for $\lambda_{a}$ and $\lambda_{b}$, and any pair of attributes whose $\lambda_{a}$ or $\lambda_{b}$ is above that threshold is considered conductive to one-level taxonomy. If such a pair is found, its contingency table is then aggregated into a two-by-two table by merging values of 
the two attributes involved. Aggregation of values should be performed in such a way that the resulting $2 \times 2$ table have the largest possible value of $\lambda$. If $\lambda$ proves sufficiently close to one, the approximate equivalence described by the $2 \times 2$ table provides a natural basis for a binary split (i.e., creation of a hierarchy element).

If an attribute is nominal, aggregation of its values is preceded by their rearrangement guided by correspondence analysis (rearrangement by correspondence analysis is a standard statistical tool: see, e.g, Mardia et al. (1979) for an illustrative example, Krzanowski (1988) for another example and a focus on singular value decomposition to replace spectral decomposition, and Krzanowski and Marriott (1994) for interpretation in terms of level profiles of attributes involved). Loosely speaking, correspondence analysis of rows of the contingency table (the same applies to columns) may enable one to rearrange the rows in such a way that the least distant (the so-called $\chi^{2}$ distance between the row profiles or, simply, the rows) rows are adjacent after rearrangement and, generally, the order in which rearranged rows appear in the table reflect the distances between the rows.

After rows and columns rearrangement, or without it if the attributes are ordered, aggregation can be easily done automatically by merging rows 1 to $a$ into one aggregated level of attribute $A$ and rows $a+1$ to $\alpha$ into another level of $A$, and merging columns 1 to $b$ into one aggregated level of attribute $B$ and columns $b+1$ to $\beta$ into another. Running over all possible pairs $(a, b)$, one finds a $2 \times 2$ table with the largest value of $\lambda$. If the maximum value of $\lambda$ is above a prespecified threshold, the table is retained for further analysis.

\section{Discretization}

There are a variety of discretization methods. Some of them are simple and obvious, such as the equal-width-intervals and equal-frequency-intervals methods. Others are not so obvious, such as ChiMerge (see Kerber, 1992), entropy-based methods (see Fayyad and Keki, 1992, 1993) and similarity based method (Van de Merckt, 1993).

Assuming that the attribute's measurements come from a continuous distribution, El-Mouadib and Koronacki (1999) proposed a discretization method which is based on the idea of building first a histogram with a bandwidth which is oversmoothed from the point of view of (asymptotic) mean integrated squared error (MISE),

$$
\operatorname{MISE}=\mathrm{E} \int_{-\infty}^{\infty}(f(x)-\hat{f}(x))^{2} d x,
$$

where $\mathrm{E}$ is the expected value, $f(\cdot)$ is the true density underlying the data and $\hat{f}(\cdot)$ denotes the histogram. See Scott (1992) for a detailed exposition; let us recall here only that, if one takes a family of all one-dimensional densities $f(\cdot)$ having the same variance, then the oversmoothed bin width can be defined as

$$
h=2.603(\mathrm{IQ}) n^{-1 / 3} \equiv \tilde{h}_{o s},
$$


where IQ is the interquartile range and $\mathrm{n}$ is the sample size (i.e., the number of measurements of the attribute being discretized).

The rationale behind the given initial choice of the bin width is simple: an oversmoothed bin width can be thought of as providing an upper bound for the discretization interval, which is then to be sought in a way consistent with the ultimate task of building a taxonomy. The final bin width or discretization interval is found by trial and error; see the iris example in El-Mouadib and Koronacki (1999).

A few remarks are in place here. First, the proposed approach is clearly ad hoc, even if natural and straightforward. However, unless some restrictions on distributions involved are imposed, no general theory, treating attributes in different scales in a unified way, is available so far (an interesting approach to discretization is given, e.g., in Ciok (1998), but there the number of clusters is assumed known a priori). Second, since partition is based on two-way tables, discretization should in fact rest on building two-dimensional histograms. And third, since simple histograms are vulnerable to bin edge problems, they should be replaced by the so-called averaged shifted histograms (ASHs). Indeed, while a simple histogram can perform well in an easy case of the famous iris data, in particular the bin edge problem can have a disastrous effect on the algorithm's ability to find clusters in not so nice data (actually, we encountered this problem in the soybean example discussed in the next section before we switched to using ASHs).

Bivariate ASHs are constructed by averaging $m^{2}$ shifted bivariate histograms, each with bins of prespecified dimension $h_{1} \times h_{2}$. Let the bin mesh of the first (in a sense the "leftmost", as we shall see) of the $\mathrm{m}^{2}$ histograms be fixed. In order to be specific, assume that the bin origin of this first histogram is the point $(0,0)$ and the histogram is built on the positive quadrant of the Cartesian plane. Then the remaining $m^{2}-1$ histograms are constructed by coordinate shifts that are multiples of $\delta_{i}=h_{i} / m, i=1,2$, and the ASH assumes the form

$$
\hat{f}(\cdot, \cdot)=\frac{1}{m^{2}} \sum_{i=1}^{m} \sum_{j=1}^{m} \hat{f}_{i j}(\cdot, \cdot),
$$

where the bin origin for the bivariate shifted histogram $\hat{f}_{i j}(\cdot, \cdot)$ is the point $\left((i-1) \delta_{1},(j-1) \delta_{2}\right)$. The given construction practically alleviates the bin edge problem (see Scott (1992) for a detailed discussion of ASHs). In our examples we begin with bin width for a given attribute equal to $\tilde{h}_{o s}$ for that attribute (admittedly, the issue of properly choosing initial dimension of bins requires an additional study).

Concluding this section, let us notice that binning of genuinely discrete data is often needed as well. Indeed, it can be advantageous to produce a histogram with a coarser, often much coarser, mesh than the original one. This can be done by trial and error, as in the handwritten digits example in El-Mouadib and Koronacki (1999). 


\section{Partition Utility and Creating Many-level Taxonomies}

In the taxonomy formation process, the search for approximate equivalence relations may result in creation of many one-level taxonomies (also called hierarchy elements by Zembowicz and Żytkow, 1996). If two such taxonomies have approximately the same data range and share common descriptors in the children classes, then the pair is merged into one one-level taxonomy. Still, after merging, several one-level taxonomies may remain. One of them is chosen as the root of multi-level taxonomy. To make that choice we use the partition utility function (see Fisher (1996), Fisher and Hapanyengwi (1993) for details). Our taxonomy formation algorithm picks the one-level taxonomy with the greatest partition utility value as the one which guides the split at this level of the tree. The partition utility function (PU) based on the Gini index is given by (analogous formula can be given for the PU function based on entropy)

$$
\begin{gathered}
P U=\frac{1}{K} \sum_{k=1}^{K} C U\left(C_{k}\right), \\
C U\left(C_{k}\right)=P\left(C_{k}\right) \sum_{i=1}^{I} \sum_{j=1}^{J_{i}}\left[P\left(A_{i}=V_{i j} \mid C_{k}\right)^{2}-P\left(A_{i}=V_{i j}\right)^{2}\right],
\end{gathered}
$$

where $K$ is the number of categories in a partition (default=2), $I$ is the number of attributes in the given data, and $J_{i}$ is the number of values of attribute $A_{i}$.

\section{Experiments and Results}

In this section, we demonstrate the results obtained by ourtaxonomy formation system for two examples. A general algorithm for building a taxonomy tree has been given by Żytkow and others and is not repeated here. To see if the algorithm works as desired, we have used real-life examples with data labeled and coming from known classes. Of course, class labels have been removed before building a taxonomy tree.

The first is the satellite data example of Mertz and Murphy (1996) from the UCI repository. We used the set of 2000 data coming from 6 classes, each with 36 attributes of continuous type. As claimed in the commentary to the repository and confirmed by principal component analysis of the data, attributes No. 17, 18, 19 and 20 contain essentially all the information hidden in the data set. Our analysis was therefore confined to this subset of attributes. The tree obtained, with discretization provided by ASHs and bin widths always equal to $\tilde{h}_{o s}$ 's, is shown in Fig.1. The split at the top level of the tree was guided by an approximate equivalence relation with $\lambda=0.882$, obtained after suitable aggregation for attributes 18 and 20, and depicted in the following $2 \times 2$ table with rows corresponding to aggregated values of attribute 18 and columns corresponding to aggregated values of attribute 20: 


\begin{tabular}{|c|c|}
\hline 9 & 1810 \\
\hline 172 & 9 \\
\hline
\end{tabular}

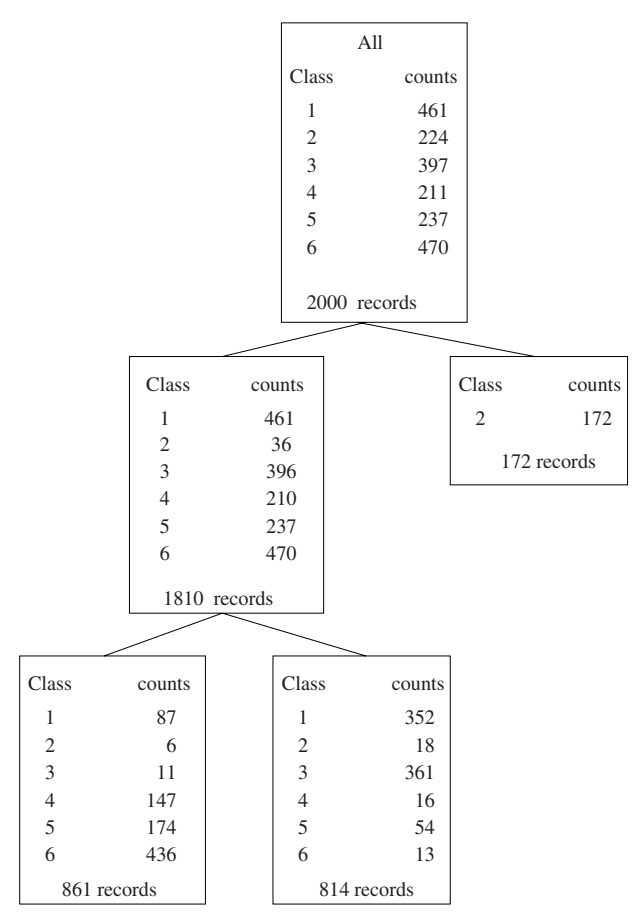

Fig. 1. Taxonomy tree for the satellite data

Of all 2000 records, 18 records which do not fit the relation, and hence do not fit the rule for the split, were "lost" from the taxonomy tree. For the lower level split, $\lambda$ was equal to 0.819 (and 135 records were "lost"). The best regularities for the two leaves had $\lambda$ 's equal, respectively, to 0.668 and 0.563 , and were therefore not used to expand the taxonomy.

The second example deals with the soybean data (again from the UCI repository). The data are nominal, with 35 attributes. The set of 307 records coming from 19 classes was chosen but all the records with missing values were excluded from the set. In this way, a subset of 266 records from 15 classes was obtained as depicted in the table below. 


\begin{tabular}{|r|l|r||r|l|c|}
\hline No. & Class description & N.of cases & No. & Class description & N.of cases \\
\hline 1 & diaporthe-stem-canker & 10 & 9 & bacterial-blight & 10 \\
2 & charcoal-rot & 10 & 10 & bacterial-pustule & 10 \\
3 & rhizoctonia-root-rot & 10 & 11 & purple-seed-stain & 10 \\
4 & phytophthora-rot & 16 & 12 & anthracnose & 20 \\
5 & brown-stem-rot & 20 & 13 & phyllosticta-leaf-spot & 10 \\
6 & powdery-mildew & 10 & 14 & alternarialeaf-spot & 40 \\
7 & downy-mildew & 10 & 15 & frog-eye-leaf-spot & 40 \\
8 & brown-spot & 40 & & & \\
\hline
\end{tabular}

The classification is shown in Fig. 2. Only 15 cases were "lost" from the taxonomy tree as violating equivalence relation in one of the $2 \times 2$ tables used to build the tree.

\section{Concluding Remarks}

In addition to the two examples from the previous section and those from ElMouadib and Koronacki (1999), the taxonomy formation system was examined on several other databases from the UCI repository. In all the examples, results were encouraging and of similar quality. While in most of the examples dendrograms based on similarity/dissimilarity measures could have been easily built, our study has confirmed the validity of the idea of using approximate equivalence relations as a basis for taxonomy formation.

\section{References}

Bishop, Y., Fienberg, S., Holland, P.: Discrete Multivariate Analysis: Theory and Practice. The MIT Press (1975)

Ciok, A.: Discretization as a total in cluster analysis. In: Rizzi, A., Vichi, M., Bock, II (eds.): Advances in Data Science and Classification Societies. Springer, (1998) 349-354

El-Mouadib, F. A., Koronacki, J.: On Taxonomy Formation by Approximate Equivalence Relations. Proc. of the 8th Workshop on Intelligent Information Systems. Polish Acad. Sci. (1999) 37-46

Fayyad, U.M., Keki, I.B.: Multi-Interval Discretization of Continuous-Valued Attributes for Classification Learning. Proc. of 13th Intern. Joint Conf. on Artificial Intelligence. Chambery, France (1993) 1022-1027

Fayyad, U.M., Keki, I.B.: Technical Note On the Handling of Continuous-Valued Attributes in Decision Tree Generation". Machine Learning 8, (1992) 87-102

Fisher, D.: Iterative Optimization and Simplification of Hierarchical Clusterings. J. of Artificial Intelligence Research (1996) 147-179

Fisher, D., Hapanyengwi, G.: Database Management and Analysis Tools of Machine Induction. Journal of Intelligent Information Systems, 2 (1993) 5-38

Goodman, L., Kruskal, W.: Measure of Association for Cross Classification. Springer Series in Statistics, Springer-Verlag, 1979. Reprinted from J. of the American Statistical Association, 49 (1954) 732-764.

Kerber, R.: ChiMerge: Discretization of Numeric Attributes. Proc. of the 10th National Conference on Artificial Intelligence, AAAI/MIT Press, (1992) 123-128 


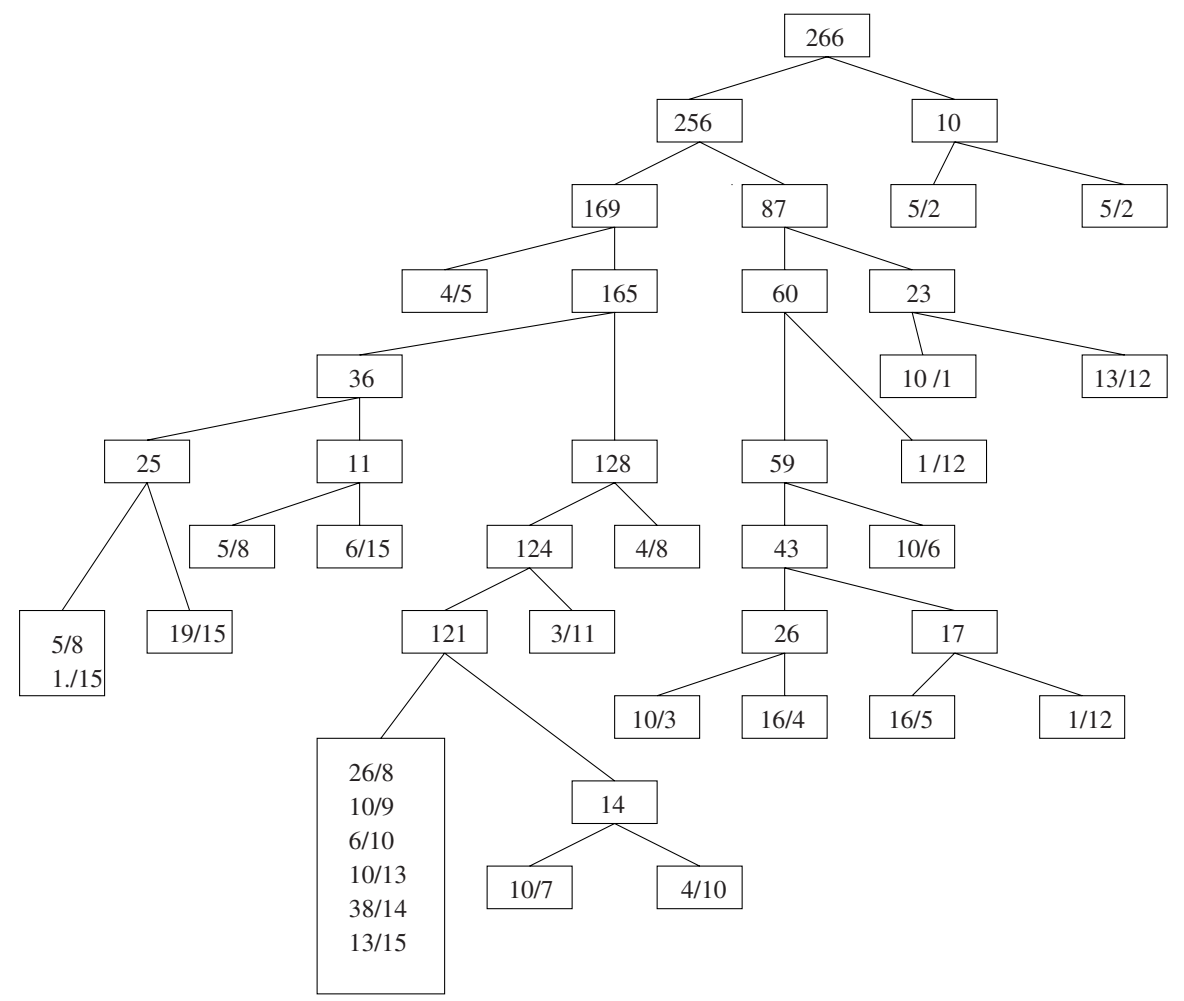

Fig. 2. Taxonomy tree for the soybean data ( $m$ in a node gives the number of records in the node and $m / n$ in leaves means " $m$ records of class $\mathrm{n}$ in the given leaf")

Krzanowski, W.: Principles of Multivariate Analysis. Oxford University Press, (1988) Krzanowski, W., Marriott, F.: Multivariate Analysis. Part 1. Edward Arnold (1994)

Mardia, K.V., Kent, J.T., Bibby, J.M.: Multivariate Analysis. Academic Press, (1979)

Merz, C.J.,Murphy, P. M.: UCI Repository of machine learning databases [http://www.ics.uci.edu/ mlearn/MLRepository.html], Irving, CA: University of California, Department of Information and Computer Science, (1996)

Scott, D.W.: Multivariate Density Estimation: Theory, Practice and Visualization. Wiley, (1992)

Troxel, M., Swarm, K., Zembowicz, R., Żytkow, J.: Concept Hierarchies: a Restricted Form of Knowledge Derived From Regularities. In: Ras, Z., Zemankova, M. (eds.): Proc. of the Seventh International Symposium on Methodologies for Intelligent Systems, (1994) 437-447

Van de Merckt, T.: Decision Trees in Numerical Attribute Space. Proc. of 13th Intern. Joint Conf. on Artificial Intelligent, Chambery, France, (1993) 1016-1021

Zembowicz, R., Żytkow, J.: From Contingency Tables to Various Forms of Knowledge in Databases. In: Fayyad, U., Piatetsky-Shapiro, G., Smyth, P., Uthurusamy, R. (eds.): Advances in Knowledge Discovery and Data Mining, AAAI Press, (1995)

Żytkow, J., Zembowicz, R.: Database Exploration in Search of Regularities. Journal of Intelligent Information Systems, 2 (1993) 39-81 